\title{
Quantum Information from Graviton-Matter Gas
}

\author{
Lukasz-Andrzej GLINKA
}

Bogoliubov Laboratory of Theoretical Physics, Joint Institute for Nuclear Research, 6 Joliot-Curie Str., 141980 Dubna, Moscow Region, Russia

E-mail: glinka@theor.jinr.ru,laglinka@gmail.com

Received May 16, 2007, in final form August 27, 2007; Published online September 04, 2007

Original article is available at http://www.emis.de/journals/SIGMA/2007/087/

\begin{abstract}
We present basics of conceptually new-type way for explaining of the origin, evolution and current physical properties of our Universe from the graviton-matter gas viewpoint. Quantization method for the Friedmann-Lemaittre Universe based on the canonical Hamilton equations of motion is proposed and quantum information theory way to physics of the Universe is showed. The current contribution from the graviton-matter gas temperature in quintessence approximation is discussed.
\end{abstract}

Key words: quantum cosmology; Friedmann Universe; nonequilibrium thermodynamics; quantum information in cosmology

2000 Mathematics Subject Classification: 83F05; 62B10; 83C45; 83C47; 82C10; 70 S05

\section{Introduction}

Origin, evolution and physical picture of our Universe are one of main areas of modern experimental as well as theoretical physics. From the theoretical physics viewpoint it seems that all known approaches, from the most popular inflationary cosmology [1] and loop quantum cosmology [2] to new alternative and compatible with experimental data conformal cosmology scenarios [3, 4, 5], produce pictures of the Universe incompatible with each other.

The most probable model for the Universe is the conformal flat Friedmann-Lemaitre spacetime. In this paper we present basics of conceptually new way for explaining the origin, evolution and current physical properties of our Universe. We found and develop the quantum information theory approach to the structure and physics of the Universe. As a model of the Universe we study the classical Friedmann-Lemaître spacetime. As the first approximation we study the quintessence model.

By the well known Dirac method [6] we construct the Hamiltonian approach to the Universe and as the solution of classical constraints we obtain the Hubble law, that confirms experimental data. According to the Dirac approach we apply the first quantization of classical primary constraints and as a result we obtain the Wheeler-DeWitt equation for the Universe. For correct quantum description of quantum gravity in this cosmological model we propose the second quantization method for the Wheeler-DeWitt equation by non-fockian distributions and by that we obtain quantum description of gravity in terms of the graviton-matter gas. By using the Bogoliubov transformation and diagonalization of equations of motion we build the correct Fock space formulation for quantum theory of the Universe. We show that a crucial role for the quantum theory of gravitation is played by quantum effective phenomena in the graviton-matter gas, which in case of the Friedmann-Lemaître spacetime are superfluidity phenomena. We find the field operator of the Universe. By using the quantum information theory methods we

${ }^{\star}$ This paper is a contribution to the Proceedings of the Seventh International Conference "Symmetry in Nonlinear Mathematical Physics" (June 24-30, 2007, Kyiv, Ukraine). The full collection is available at http://www.emis.de/journals/SIGMA/symmetry2007.html 
calculate the density matrix and entropy of the graviton-quintessence gas. We predict a relation between temperature and number of particles, and evaluate equation of state for the gas.

In this paper we will use cosmological units system $\hbar=c=k_{B}=\frac{8 \pi G}{3}=1$, where $\hbar$ is the Dirac constant, $c$ is the velocity of light, $k_{B}$ is the Boltzmann constant, $e$ is the elementary charge and $G$ is the Newton gravitational constant.

\section{Classical Friedmann-Lemaître spacetime}

\subsection{Conformal flat metric}

As a cosmological model of the Universe we will study an exact solution of the Einstein field equations of general relativity [7], homogenous, flat and isotropic expanding or contracting spacetime founded and studied by A.A. Friedmann [8] in the Einstein field equations context and by G.-H. Lemaître [9] in the Big Bang theory as the origin of the Universe context. This model is characterized by the interval

$$
d s^{2}=d t d t-a^{2}(t) d x^{i} d x^{i},
$$

where $a(t)$ is known as the Friedmann conformal scale factor. The spacetime interval (2.1) can be transformed to conformal flat form by diffeomorphism of $t$. Friedmann [8] introduced change of time $t$ on the conformal time $\eta$ by formula

$$
\eta=\int_{t_{0}}^{t} \frac{d t^{\prime}}{a\left(t^{\prime}\right)}
$$

This is a replacement of two diffeoinvariant times $t \rightarrow \eta$. With using of the conformal time (2.2) the interval (2.1) reduces into the pseudo-Euclidean form

$$
d s^{2}=a^{2}(\eta)\left(d \eta d \eta-d x^{i} d x^{i}\right) .
$$

Conceptually new moment in the general relativity was introducing by P.A.M. Dirac [6] of the lapse function $N_{d}\left(x^{0}\right)$ defined by the formula

$$
d \eta=N_{d}\left(x^{0}\right) d x^{0},
$$

where $x^{0}$ is time-coordinate as object of diffeomorphisms

$$
x^{0} \rightarrow \widetilde{x}^{0}=\widetilde{x}\left(x^{0}\right),
$$

introduced by Albert Einstein [7] in the general relativity context and developed by A.L. Zelmanov [10] in cosmology context and by B.M. Barbashov et al. [11] in diffeoinvariant Hamiltonian cosmological perturbation theory.

\subsection{The Dirac Hamiltonian approach}

Recall that the Einstein-Hilbert general relativity with presence of the matter fields is described by the action found by David Hilbert [12]

$$
\mathcal{A}=\int d^{4} x \sqrt{-g}\left\{-\frac{1}{6} \mathcal{R}+\mathcal{L}_{M}\right\}
$$

where $g=\operatorname{det} g_{\mu \nu}, g_{\mu \nu}$ is the metric tensor of the spacetime, $\mathcal{L}_{M}$ is the matter field Lagrangian and $\mathcal{R}$ is the Ricci scalar (see for example $[13,14]$ ). In cosmological considerations the Lagrangian $\mathcal{L}_{M}$ describes matter in the Universe. As the Universe is classical object, the matter is characterized by mean-field properties. 
The Hilbert action (2.4) calculated for the Friedmann-Lemaitre metric (2.1) is

$$
\mathcal{A}[a]=-V \int d x^{0}\left\{\frac{1}{N_{d}}\left(\frac{d a}{d x^{0}}\right)^{2}+N_{d} a^{4}\left\langle\mathcal{H}\left(x^{0}\right)\right\rangle\right\},
$$

where

$$
\left\langle\mathcal{H}\left(x^{0}\right)\right\rangle=\frac{1}{V} \int d^{3} x \mathcal{H}_{M}\left(x^{i}, x^{0}\right), \quad V=\int d^{3} x<\infty,
$$

are the zeroth Fourier harmonic of the matter Hamiltonian and spatial volume, respectively. We apply for the action (2.5) the Hamiltonian reduction procedure. Firstly, we calculate the canonical conjugate momentum corresponding to this action

$$
p_{a}=-\frac{2 V}{N_{d}} \frac{d a}{d x^{0}}
$$

and with use of this momentum the action (2.5) becomes

$$
\mathcal{A}[a]=-V \int d x^{0}\left\{\frac{p_{a}^{2}}{4 V^{2}}+a^{4}\left\langle\mathcal{H}\left(x^{0}\right)\right\rangle\right\}
$$

From the Hamiltonian reduction viewpoint the reduced action has a form

$$
\mathcal{A}[a]=\int d x^{0}\left\{p_{a} \frac{d a}{d x^{0}}-\mathrm{H}\left(p_{a}, a\right)\right\}
$$

where the Hamiltonian $\mathrm{H}\left(p_{a}, a\right)$. By this we obtain

$$
\mathrm{H}\left(p_{a}, a\right)=N_{d}\left[-\frac{p_{a}^{2}}{4 V}+V\left\langle\mathcal{H}\left(x^{0}\right)\right\rangle a^{4}\right]
$$

According to the Dirac approach the action principle with respect to the lapse function $N_{d}$ applied to the action (2.7) produces Hamiltonian constraint equation. In the considered case we have the constraint equation

$$
\frac{\delta \mathcal{A}[a]}{\delta N_{d}}=0=-\frac{p_{a}^{2}}{4 V}+V\left\langle\mathcal{H}\left(x^{0}\right)\right\rangle a^{4} .
$$

We can resolve this classical constraint equation immediately. As a result, we obtain

$$
\frac{a(t)}{a\left(t_{0}\right)}=\exp \left\{\operatorname{sgn}\left(t-t_{0}\right) \int_{t_{0}}^{t} N_{d}\left(x^{0}\right) d x^{0} \sqrt{\left\langle\mathcal{H}\left(x^{0}\right)\right\rangle}\right\},
$$

and it is the Hubble law.

From the other side the constraint equation (2.8) expressed in the Dirac conformal time has a solution

$$
p_{a}=-2 V \frac{d a}{d \eta}= \pm \omega_{a},
$$

and defines values of the canonical conjugate momentum (2.6). In (2.9) the quantity $\omega_{a}$ is time diffeoinvariant variable

$$
\omega_{a}=2 V \sqrt{\langle\mathcal{H}(\eta)\rangle} a^{2}(\eta) .
$$


Equation (2.9) produces the ordinary differential equation on $a(\eta)$

$$
-\frac{d a}{d \eta}= \pm \sqrt{\langle\mathcal{H}(\eta)\rangle} a^{2}(\eta)
$$

In this equation variables can be separated immediately and elementary integration leads to the result

$$
a(\eta)=\frac{a\left(\eta_{0}\right)}{1+z\left(\eta_{0} ; \eta\right)},
$$

where we have defined the quantity

$$
z\left(\eta_{0} ; \eta\right)=a\left(\eta_{0}\right) \operatorname{sgn}\left(\eta-\eta_{0}\right) \int_{\eta_{0}}^{\eta} d \eta^{\prime} \sqrt{\left\langle\mathcal{H}\left(\eta^{\prime}\right)\right\rangle}
$$

The nature of $z\left(\eta_{0}, \eta\right)$ can be understood if we rewrite $(2.12)$ in the power series form [14, 15]

$$
z\left(\eta_{0} ; \eta\right)=H_{0}\left(\eta-\eta_{0}\right)+\left(1+\frac{q_{0}}{2}\right) H_{0}^{2}\left(\eta-\eta_{0}\right)^{2}+\cdots .
$$

The quantity $z\left(\eta_{0}, \eta\right)$ is nothing else than the redshift. The constants $H_{0}$ and $q_{0}$ are called the Hubble parameter and the deceleration parameter

$$
\begin{aligned}
& H_{0}=\sqrt{\left\langle\mathcal{H}\left(\eta_{0}\right)\right\rangle} a\left(\eta_{0}\right), \\
& q_{0}=\frac{2}{H_{0}} \frac{\left\langle\dot{\mathcal{H}}\left(\eta_{0}\right)\right\rangle}{\left\langle\mathcal{H}\left(\eta_{0}\right)\right\rangle}-2 .
\end{aligned}
$$

The result (2.11) lies in agreement with experimental data, this formula describes the Hubble law. It is clear that the Hubble parameter (2.13) and the deceleration parameter (2.14) are diffeoinvariants.

\subsection{Quintessence}

We understand the quintessence as a kind of matter characterized by constant energy - the cosmological constant $\Lambda$. The cosmological constant is equal to zeroth mode of the matter Hamiltonian. By this way properties of the constant $\Lambda$ are

$$
\langle\mathcal{H}(\eta)\rangle=\left\langle\mathcal{H}\left(\eta_{0}\right)\right\rangle=\Lambda, \quad\langle\dot{\mathcal{H}}(\eta)\rangle=\left\langle\dot{\mathcal{H}}\left(\eta_{0}\right)\right\rangle=0 .
$$

In this approximation the Hubble constant (2.13) and the deceleration parameter (2.14) have a simple form

$$
H_{0}=\Lambda^{1 / 2} a\left(\eta_{0}\right), \quad q_{0}=-2,
$$

and the redshift is

$$
z\left(\eta_{0} ; \eta\right)=H_{0}\left|\eta-\eta_{0}\right| .
$$

The solution of classical constraints (2.10) for the quintessence has a form

$$
p_{a}= \pm \omega_{a}(\eta)= \pm 2 V \Lambda^{1 / 2} a^{2}= \pm \omega_{a}\left(\eta_{0}\right)\left(\frac{a(\eta)}{a\left(\eta_{0}\right)}\right)^{2}
$$

where

$$
\omega_{a}\left(\eta_{0}\right)=2 V \Lambda^{1 / 2} a^{2}\left(\eta_{0}\right)=2 V \frac{H_{0}^{2}}{\sqrt{\Lambda}},
$$

is diffeoinvariant constant. 


\section{Quantum gravity and collective phenomena}

In this section we quantize the Friedmann-Lemaître spacetime with quintessence. In contrast to hitherto existing approaches we propose the procedure based on the Hamilton equations of motion

1. By first quantization of classical constraints we obtain the Wheeler-DeWitt equation for the wave function $\Psi$ of the Universe,

2. We treat the wave function $\Psi$ as a classical field, and we construct classical field theory the canonical Hamilton equations,

3. We quantize the canonical Hamilton equations by non-fockian distributions in the Fock space of annihilation and creation operators,

4. We apply the Bogoliubov transformation and by diagonalization of the quantized canonical Hamilton equations in the Fock space we carry evolution from operators onto the Bogoliubov coefficients,

5. We find the field operator $\Psi$ of the Universe and conjugate momentum operator $\Pi_{\Psi}$.

\subsection{Quantum mechanics of the Universe}

In agreement with P.A.M. Dirac [6] we apply the first quantization of the classical constraint equation. Recall that for the Friedmann-Lemaitre Universe the Hamiltonian constraint equation has a form

$$
p_{a}^{2}-\omega_{a}^{2}=0,
$$

with $\omega_{a}$ given by (2.10). Classical solution of this constraint equation is given by the Hubble law (2.11) with the redshift (2.15).

The first quantization of the Hamiltonian constraint equation is given by canonical commutation relation in the standard form

$$
i\left[\hat{\mathrm{p}}_{a}, a\right]=1,
$$

where $\hat{\mathrm{p}}_{a}=-i \frac{\partial}{\partial a}$ is the momentum operator corresponding to canonical conjugate momentum $p_{a}$. We assume that the wave function $\Psi(a)$ for quantum theory exists. The final result of this step is the quantum evolution equation

$$
\left(\partial_{a} \partial_{a}+\omega_{a}^{2}\right) \Psi(a)=0,
$$

which is known as the Wheeler-DeWitt equation [16, 17]. This equation defines quantum mechanics description of the spacetime. Classical solutions of (3.2) fulfill all conditions for wave function.

\subsection{Classical field theory of the Universe}

The equation (3.2) looks like the Klein-Gordon equation [18] for the boson with mass $\omega_{a}$. Let us consider the Wheeler-DeWitt equation as an equation of motion for the classical field $\Psi$ and describe the Hamiltonian classical field theory of $\Psi$. For this purpose we should construct the classical action which produces the equation of motion (3.2) from the Hamilton action principle. The correct form of the classical action can be obtained by heuristic analogy with the KleinGordon case

$$
\mathcal{S}[\Psi]=\frac{1}{2} \int_{a\left(\eta_{0}\right)}^{a(\eta)} d a\left\{\left(\partial_{a} \Psi\right)^{2}-\omega_{a}^{2} \Psi^{2}\right\} .
$$


Let us check that this action produces the Wheeler-DeWitt equation. The Hamilton action principle gives

$$
\begin{aligned}
\delta \mathcal{S}[\Psi] \equiv 0 & =\frac{\delta \mathcal{S}[\Psi]}{\delta \Psi} \delta \Psi+\frac{\delta \mathcal{S}[\Psi]}{\delta \partial_{a} \Psi} \delta \partial_{a} \Psi=\frac{\delta \mathcal{S}[\Psi]}{\delta \Psi} \delta \Psi+\frac{\delta \mathcal{S}[\Psi]}{\delta \partial_{a} \Psi} \partial_{a} \delta \Psi \\
& =\left\{\frac{\delta \mathcal{S}[\Psi]}{\delta \Psi}-\partial_{a} \frac{\delta \mathcal{S}[\Psi]}{\delta \partial_{a} \Psi}\right\} \delta \Psi+\partial_{a}\left\{\frac{\delta \mathcal{S}[\Psi]}{\delta \partial_{a} \Psi} \delta \Psi\right\} .
\end{aligned}
$$

The second term vanishes on boundaries and thus we obtain

$$
\frac{\delta \mathcal{S}[\Psi]}{\delta \Psi}-\partial_{a} \frac{\delta \mathcal{S}[\Psi]}{\delta \partial_{a} \Psi}=0,
$$

or after using (3.3)

$$
\int d a\left\{\omega_{a}^{2} \Psi+\partial_{a} \partial_{a} \Psi\right\}=0 \Rightarrow\left(\partial_{a} \partial_{a}+\omega_{a}^{2}\right) \Psi=0,
$$

what is exactly the Wheeler-DeWitt equation (3.2) for the field $\Psi$. By this the Wheeler-DeWitt equation is an equation of motion for the classical field $\Psi$ and the heuristic action (3.3) is correct.

Let us calculate the canonical conjugate momentum field corresponding to the action (3.3)

$$
\Pi_{\Psi}=\frac{\delta \mathcal{S}[\Psi]}{\delta\left(\partial_{a} \Psi\right)}=\partial_{a} \Psi
$$

With this momentum the action (3.3) reduces into the form

$$
\mathcal{S}[\Psi]=\int d a\left\{\Pi_{\Psi} \partial_{a} \Psi-\mathrm{H}\left(\Pi_{\Psi}, \Psi\right)\right\},
$$

where

$$
\mathrm{H}\left(\Pi_{\Psi}, \Psi\right)=\frac{1}{2}\left(\Pi_{\Psi}^{2}+\omega_{a}^{2} \Psi^{2}\right),
$$

is the Hamiltonian that describes evolution of classical field $\Psi$. The canonical Hamilton equations of motion for the classical field theory described by the Hamiltonian (3.6) are as follows

$$
\begin{aligned}
& \frac{\partial \mathrm{H}\left(\Pi_{\Psi}, \Psi\right)}{\partial \Pi_{\Psi}}=\partial_{a} \Psi, \\
& -\frac{\partial \mathrm{H}\left(\Pi_{\Psi}, \Psi\right)}{\partial \Psi}=\partial_{a} \Pi_{\Psi},
\end{aligned}
$$

after calculations we can rewrite these equations in the form

$$
\partial_{a}\left[\begin{array}{c}
\Psi \\
\Pi_{\Psi}
\end{array}\right]=\left[\begin{array}{cc}
0 & 1 \\
-\omega_{a}^{2} & 0
\end{array}\right]\left[\begin{array}{c}
\Psi \\
\Pi_{\Psi}
\end{array}\right] .
$$

The equation (3.7) leads to the relation (3.4), and the equation (3.8) is equivalent to the WheelerDeWitt equation (3.2) after using the equation (3.7). Hitherto existing approaches to quantization problem of the Friedmann-Lemaitre spacetime, and generally to quantization problem for gravity, was based on testing of solution or the second quantization of the Wheeler-DeWitt equation for the theory. As opposed to these approaches, we will base the quantum theory of gravity on the canonical Hamilton equations of motion. 


\subsection{Quantization of the Hamilton equations of motion}

The analogy method presented in previous subsection produces conclusion that given system is a boson and by this the corresponding quantum field theory description should be build in boson type Fock space language. The boson type Fock space of creation $\mathcal{G}^{\dagger}$ and annihilation $\mathcal{G}$ operators are standard, constructed by the canonical commutation relations $[18,19,20]$

$$
\begin{aligned}
& {\left[\mathcal{G}(a(\eta)), \mathcal{G}^{\dagger}\left(a\left(\eta^{\prime}\right)\right)\right]=\delta\left(a(\eta)-a\left(\eta^{\prime}\right)\right),} \\
& {\left[\mathcal{G}(a(\eta)), \mathcal{G}\left(a\left(\eta^{\prime}\right)\right)\right]=0 .}
\end{aligned}
$$

By way of analogy with the Klein-Gordon theory we propose the following second quantization by the non-fockian type distributions in the Fock space $(a \equiv a(\eta))$

$$
\begin{aligned}
& \Psi(a)=\frac{1}{\sqrt{2 \omega_{a}}}\left(\mathcal{G}(a)+\mathcal{G}^{\dagger}(a)\right), \\
& \Pi_{\Psi}(a)=-i \sqrt{\frac{\omega_{a}}{2}}\left(\mathcal{G}(a)-\mathcal{G}^{\dagger}(a)\right),
\end{aligned}
$$

or in compact form

$$
\left[\begin{array}{c}
\Psi \\
\Pi_{\Psi}
\end{array}\right]=\left[\begin{array}{cc}
\frac{1}{\sqrt{2 \omega_{a}}} & \frac{1}{\sqrt{2 \omega_{a}}} \\
-i \sqrt{\frac{\omega_{a}}{2}} & i \sqrt{\frac{\omega_{a}}{2}}
\end{array}\right]\left[\begin{array}{c}
\mathcal{G} \\
\mathcal{G}^{\dagger}
\end{array}\right]
$$

The correct canonical commutation relation for the field operators $\Psi$ and $\Pi_{\Psi}$

$$
\left[\Psi\left(a\left(\eta^{\prime}\right)\right), \Pi_{\Psi}(a(\eta))\right]=i \delta\left(a(\eta)-a\left(\eta^{\prime}\right)\right)
$$

is preserved automatically. The distributions (3.10) and (3.11) contain the new element that will play a crucial role - the normalization coefficients depend on $a$. It causes that (3.10) and (3.11) are nonfockian representations in the Fock space of the system.

Using the non-fockian distributions (3.10) and (3.11) we can translate the Wheeler-DeWitt action (3.5) into the Fock space language

$$
\mathcal{S}\left(\mathcal{G}, \mathcal{G}^{\dagger}\right)=\int \mathcal{D} a\left\{i \frac{\mathcal{G}^{\dagger} \partial_{a} \mathcal{G}-\mathcal{G} \partial_{a} \mathcal{G}^{\dagger}}{2}-\mathcal{H}\right\}
$$

where we have used the Feynman-type measure, and the effective Hamiltonian $\mathcal{H}$ is equal to

$$
\mathcal{H}=\left(\mathcal{G}^{\dagger} \mathcal{G}+\frac{1}{2}\right) \omega_{a}+\frac{i}{2}\left(\mathcal{G}^{\dagger} \mathcal{G}^{\dagger}-\mathcal{G} \mathcal{G}\right) \Delta
$$

where $\Delta=\frac{\partial_{a} \omega_{a}}{2 \omega_{a}}$ has the meaning of coupling. The Hamiltonian (3.13) is well known from the many particle theories as the Hamiltonian describing the boson superfluidity phenomenon. By this way the coupling $\Delta$ manifests collective phenomena. The superfluidity in quantum cosmology was first discussed in paper [21].

\subsection{Diagonalization of equations of motion}

By quantization of the canonical Hamilton equations of motion (3.9) we obtain the equations of motion for the creation and annihilation operators in the Fock space

$$
i \partial_{a}\left[\begin{array}{c}
\mathcal{G} \\
\mathcal{G}^{\dagger}
\end{array}\right]=\left[\begin{array}{cc}
-\omega_{a} & 2 i \Delta \\
2 i \Delta & \omega_{a}
\end{array}\right]\left[\begin{array}{c}
\mathcal{G} \\
\mathcal{G}^{\dagger}
\end{array}\right] .
$$


These equations are understood as the Heisenberg equations for $\mathcal{G}$ and $\mathcal{G}^{\dagger}$ [19] with nonlinearity in form of nondiagonal elements in the evolution matrix (3.14). We see that the quantum evolution (3.14) is not diagonal. Now we must diagonalize this evolution. Firstly we use

The boson Bogoliubov transformation. We change the basis $\left(\mathcal{G}^{\dagger}, \mathcal{G}\right)$ to another basis $\left(\mathcal{W}^{\dagger}, \mathcal{W}\right)$ in the Fock space by the general transformation $(a \equiv a(\eta))$

$$
\left[\begin{array}{c}
\mathcal{W}(a) \\
\mathcal{W}^{\dagger}(a)
\end{array}\right]=\left[\begin{array}{cc}
u(a) & v(a) \\
v^{*}(a) & u^{*}(a)
\end{array}\right]\left[\begin{array}{c}
\mathcal{G}(a) \\
\mathcal{G}^{\dagger}(a)
\end{array}\right]
$$

If we want to preserve the canonical commutation relations in the basis $\left(\mathcal{W}^{\dagger}, \mathcal{W}\right)$

$$
\left[\mathcal{W}(a(\eta)), \mathcal{W}^{\dagger}\left(a\left(\eta^{\prime}\right)\right)\right]=\delta\left(a(\eta)-a\left(\eta^{\prime}\right)\right), \quad\left[\mathcal{W}(a(\eta)), \mathcal{W}\left(a\left(\eta^{\prime}\right)\right)\right]=0
$$

we obtain the rotation condition

$$
|u(a)|^{2}-|v(a)|^{2}=1 .
$$

After this we apply

Diagonalization of quantum canonical Hamilton equations of motion. The $a$ evolution in the basis $\left(\mathcal{G}^{\dagger}, \mathcal{G}\right)(3.14)$ is transformed into the evolution in the basis $\left(\mathcal{W}^{\dagger}, \mathcal{W}\right)$ in the form

$$
i \partial_{a}\left[\begin{array}{c}
\mathcal{W} \\
\mathcal{W}^{\dagger}
\end{array}\right]=\left[\begin{array}{cc}
\omega_{1} & 0 \\
0 & \omega_{2}
\end{array}\right]\left[\begin{array}{c}
\mathcal{W} \\
\mathcal{W}^{\dagger}
\end{array}\right]
$$

with some diagonalization energies $\omega_{1}$ and $\omega_{2}$. In this way there is no coupling in the basis $\left(\mathcal{W}^{\dagger}, \mathcal{W}\right)$

This procedure produces equations for the Bogoliubov coefficients $u$ and $v$

$$
i \partial_{a}\left[\begin{array}{l}
v \\
u
\end{array}\right]=\left[\begin{array}{cc}
-\omega_{a} & -2 i \Delta \\
-2 i \Delta & \omega_{a}
\end{array}\right]\left[\begin{array}{l}
v \\
u
\end{array}\right]
$$

and values of the diagonalization energies $\omega_{1}$ and $\omega_{2}$ are

$$
\omega_{1}=\omega_{2}=0 .
$$

By this we have solution of the equations (3.16)

$$
\mathcal{W}(a)=\mathcal{W}\left(a_{0}\right), \quad \mathcal{W}^{\dagger}(a)=\mathcal{W}^{\dagger}\left(a_{0}\right),
$$

and we can see that the operator $\mathcal{N}_{\mathcal{W}}=\mathcal{W}^{\dagger} \mathcal{W}=\mathcal{W}^{\dagger}\left(a_{0}\right) \mathcal{W}\left(a_{0}\right)$ is an integral of motion

$$
\partial_{a} \mathcal{N}_{\mathcal{W}}=0
$$

By this the stable Bogoliubov vacuum state $|0\rangle$ exists

$$
\mathcal{W}|0\rangle=0, \quad\langle 0| \mathcal{W}^{\dagger}=0 .
$$

Since the hyperbolic identity (3.15), the Bogoliubov coefficients $u$ and $v$ can be parameterized as $[22]$

$$
v(a)=e^{i \theta(a)} \sinh \phi(a), \quad u(a)=e^{i \theta(a)} \cosh \phi(a),
$$

and thus the equations (3.17) are equivalent to the equations

$$
\partial_{a} \theta(a)= \pm \omega_{a}=p_{a}, \quad \partial_{a} \phi(a)=-2 \Delta=-\frac{\partial_{a} \omega}{\omega}=-\partial_{a} \ln \left|\omega_{a}\right|,
$$


with obvious solutions

$$
\theta(a)=\int_{a_{0}}^{a} p_{a} d a, \quad \phi(a)=-\ln \left|\frac{\omega_{a}(\eta)}{\omega_{a}\left(\eta_{0}\right)}\right| .
$$

By this we have

$$
\begin{aligned}
& v(a)=\frac{1}{2} \exp \left\{i \int_{a_{0}}^{a} p_{a} d a\right\}\left(\frac{\omega_{a}\left(\eta_{0}\right)}{\omega_{a}(\eta)}-\frac{\omega_{a}(\eta)}{\omega_{a}\left(\eta_{0}\right)}\right), \\
& u(a)=\frac{1}{2} \exp \left\{i \int_{a_{0}}^{a} p_{a} d a\right\}\left(\frac{\omega_{a}\left(\eta_{0}\right)}{\omega_{a}(\eta)}+\frac{\omega_{a}(\eta)}{\omega_{a}\left(\eta_{0}\right)}\right) .
\end{aligned}
$$

In the Einstein-Hilbert theory, gravitation does not exist without structure of spacetime - the spacetime creates gravitation, and gravitation creates the spacetime. The formalism presented here describes the spacetime, which in our problem is the Friedmann-Lemaitre Universe, in the language of collective phenomena. In this formulation, these collective phenomena take place in gas, which is a nontrivial mixture of quanta of gravity and the quintessence, that is a model approximation of bosons and fermions fields. Generally our proposition is based on applying of the graviton-matter gas approach to quantization of gravity with matter fields presence. In this language we will formulate physics of the Universe.

\subsection{Field operator of the Universe}

As we have seen, the second quantization of the canonical Hamilton equations of motion (3.9) really represents classical field theory phase space $\left[\Psi(a) \Pi_{\Psi}(a)\right]^{T}$ by the non-fockian representation (3.12) in the Fock space with using of the correct Bogoliubov transformed basis

$$
\left[\begin{array}{c}
\mathcal{G}(a) \\
\mathcal{G}^{\dagger}(a)
\end{array}\right]=\left[\begin{array}{cc}
u^{*}(a) & -v(a) \\
-v^{*}(a) & u(a)
\end{array}\right]\left[\begin{array}{c}
\mathcal{W}\left(a_{0}\right) \\
\mathcal{W}^{\dagger}\left(a_{0}\right)
\end{array}\right]
$$

This procedure produces quantum field theory phase space described by general relation

$$
\left[\begin{array}{c}
\Psi(a) \\
\Pi_{\Psi}(a)
\end{array}\right]=\left[\begin{array}{cc}
\frac{u^{*}(a)-v^{*}(a)}{\sqrt{2 \omega_{a}}} & \frac{u(a)-v(a)}{\sqrt{2 \omega_{a}}} \\
-i \sqrt{\frac{\omega_{a}}{2}}\left(u^{*}(a)+v^{*}(a)\right) & i \sqrt{\frac{\omega_{a}}{2}}(u(a)+v(a))
\end{array}\right]\left[\begin{array}{c}
\mathcal{W}\left(a_{0}\right) \\
\mathcal{W}^{\dagger}\left(a_{0}\right)
\end{array}\right],
$$

or after calculations

$$
\left[\begin{array}{c}
\Psi(a) \\
\Pi_{\Psi}(a)
\end{array}\right]=\left[\begin{array}{cc}
\frac{1}{\omega_{a}\left(\eta_{0}\right)} \sqrt{\frac{\left|\partial_{a} \theta\right|}{2}} e^{-i \theta} & \frac{1}{\omega_{a}\left(\eta_{0}\right)} \sqrt{\frac{\left|\partial_{a} \theta\right|}{2}} e^{i \theta} \\
-i \omega_{a}\left(\eta_{0}\right) \sqrt{\frac{1}{2\left|\partial_{a} \theta\right|}} e^{-i \theta} & i \omega_{a}\left(\eta_{0}\right) \sqrt{\frac{1}{2\left|\partial_{a} \theta\right|}} e^{i \theta}
\end{array}\right]\left[\begin{array}{c}
\mathcal{W}\left(a_{0}\right) \\
\mathcal{W}^{\dagger}\left(a_{0}\right)
\end{array}\right]
$$

where

$$
\partial_{a} \theta(a)= \pm \omega_{a}\left(\eta_{0}\right)\left(\frac{a(\eta)}{a\left(\eta_{0}\right)}\right)^{2}, \quad \theta(a)=\int_{a_{0}}^{a} p_{a} d a .
$$

By this we have the field operator of the Universe

$$
\Psi(a)=\frac{1}{\omega_{a}\left(\eta_{0}\right)} \sqrt{\frac{\left|\partial_{a} \theta\right|}{2}}\left(e^{i \theta} \mathcal{W}^{\dagger}\left(a_{0}\right)+e^{-i \theta} \mathcal{W}\left(a_{0}\right)\right),
$$

and similarly we can read the momentum field

$$
\Pi_{\Psi}(a)=i \omega_{a}\left(\eta_{0}\right) \sqrt{\frac{1}{2\left|\partial_{a} \theta\right|}}\left(e^{i \theta} \mathcal{W}^{\dagger}\left(a_{0}\right)-e^{-i \theta} \mathcal{W}\left(a_{0}\right)\right) .
$$




\section{Thermodynamics of the Universe}

\subsection{Density matrix and entropy}

Obviously, the graviton-matter gas is an open quantum system [23] and should be described by nonequilibrium quantum statistical mechanics methods [24]. In the standard approach to nonequilibrium processes the one-particle density operator is the particle number operator. In the case of the graviton-matter gas a role of particles is played by elements of this gas. By this, the density operator for the system is

$$
\varrho_{\mathcal{G}}=\mathcal{G}^{\dagger} \mathcal{G}
$$

and if we rewrite this operator in $\left(\mathcal{W}, \mathcal{W}^{\dagger}\right)$ basis we have

$$
\varrho_{\mathcal{G}}=\mathrm{W}^{\dagger} \rho \mathrm{W}
$$

where $\mathbf{W}=\left[\begin{array}{c}\mathcal{W} \\ \mathcal{W}^{\dagger}\end{array}\right]$ and

$$
\rho=\left[\begin{array}{cc}
|u|^{2} & -u v \\
-u^{*} v^{*} & |v|^{2}
\end{array}\right]
$$

is the density matrix for graviton-matter gas in thermodynamical equilibrium.

Physical entropy of the system is defined by the formula well known from the quantum information theory [25]

$$
\mathrm{S}=-\frac{\operatorname{tr}(\rho \ln \rho)}{\operatorname{tr}(\rho)} \equiv \ln \Omega
$$

where $\Omega$ is the partition function that for the graviton-quintessence gas is equal to

$$
\Omega=\frac{1}{2|u|^{2}-1} .
$$

\subsection{Temperature}

In case of the graviton-matter gas we have thermodynamical nonequilibrium, particles of the gas go out from the system. As a result we have diagonalized equations of motion, and we have found basis where particle number operator is an integral of motion and thus in this basis we have thermodynamical equilibrium of the graviton-matter gas. So we can use equilibrium statistical mechanics formulas for thermodynamical description of the system.

If we identify the partition function of the graviton-quintessence gas (4.1) with the BoseEinstein type partition function we obtain

$$
\Omega=\frac{1}{2|u|^{2}-1} \equiv \frac{1}{\exp \frac{\mathrm{E}}{\mathrm{T}}-1} \Longrightarrow \mathrm{T}=\frac{\mathrm{E}}{\ln 2|u|^{2}},
$$

where we used the Gibbs state type. This type of identification has a meaning if and only if we identify

$$
\mathrm{E} \equiv \mathrm{U}-\mu \mathrm{N}
$$

where $\mathrm{U}$ is internal energy, $\mu$ is chemical potential and $\mathrm{N}$ is number of particles of the gravitonmatter gas, respectively. 
As we have seen, the Hamiltonian describes considered system was given by (3.13)

$$
\mathcal{H}=\left(\mathcal{G}^{\dagger} \mathcal{G}+\frac{1}{2}\right) \omega_{a}+\frac{i}{2}\left(\mathcal{G}^{\dagger} \mathcal{G}^{\dagger}-\mathcal{G G}\right) \Delta
$$

In a diagonalized basis this effective Hamiltonian has a form

$$
\mathcal{H}=\mathrm{W}^{\dagger} \mathbf{H W}
$$

where

$$
\mathbf{H}=\left[\begin{array}{cc}
\frac{|u|^{2}+|v|^{2}}{2} \omega_{a}+i \frac{u^{*} v-u v^{*}}{2} \Delta & -u v \omega_{a}+i \frac{u u-v v}{2} \Delta \\
-u^{*} v^{*} \omega_{a}+i \frac{v^{*} v^{*}-u^{*} u^{*}}{2} \Delta & \frac{|u|^{2}+|v|^{2}}{2} \omega_{a}+i \frac{u^{*} v-u v^{*}}{2} \Delta
\end{array}\right],
$$

is the matrix of the effective Hamiltonian.

In the quantum statistical mechanics [24] internal energy $U$ of thermodynamical system is defined by quantum mechanical average Hamiltonian of the thermodynamical system

$$
\mathrm{U}=\langle\mathbf{H}\rangle=\frac{\operatorname{tr}(\rho \mathbf{H})}{\operatorname{tr} \rho} .
$$

After averaging we obtain

$$
\mathrm{U}=\left(\frac{1}{2}+2 \mathrm{~N}+\frac{\mathrm{N}}{2 \mathrm{~N}+1}\right)(\sqrt{\mathrm{N}+1}-\sqrt{\mathrm{N}}) \omega_{a}\left(\eta_{0}\right)
$$

where $\mathrm{N}$ is a number of particles of the gas

$$
\mathrm{N}=|v|^{2} .
$$

So the chemical potential for the gas is

$$
\mu=\left[2+\frac{1}{(2 \mathrm{~N}+1)^{2}}-\frac{\frac{1}{2}+2 \mathrm{~N}+\frac{\mathrm{N}}{2 \mathrm{~N}+1}}{2 \sqrt{\mathrm{N}(\mathrm{N}+1)}}\right](\sqrt{\mathrm{N}+1}-\sqrt{\mathrm{N}}) \omega_{a}\left(\eta_{0}\right),
$$

and temperature T (see Fig. 1) is equal to

$$
\mathrm{T}=\frac{\sqrt{\mathrm{N}+1}-\sqrt{\mathrm{N}}}{\ln (2 \mathrm{~N}+2)}\left[\left(\frac{1}{2}+2 \mathrm{~N}+\frac{\mathrm{N}}{2 \mathrm{~N}+1}\right)\left(1+\frac{1}{2} \sqrt{\frac{\mathrm{N}}{\mathrm{N}+1}}\right)-2 \mathrm{~N}-\frac{\mathrm{N}}{(2 \mathrm{~N}+1)^{2}}\right] \omega_{a}\left(\eta_{0}\right) .
$$

We see that now $(\mathrm{N}=0)$ we have a finite contribution from the gas temperature

$$
\mathrm{T}[0]=\frac{\omega_{a}\left(\eta_{0}\right)}{\ln 4} .
$$

Now we can conclude that the equation of state for the graviton-matter gas in case of quintessence is equal to

$$
\frac{\mathrm{U}}{\mathrm{T}}=\frac{\ln (2 \mathrm{~N}+2)}{1+\frac{1}{2} \sqrt{\frac{\mathrm{N}}{\mathrm{N}+1}}-\frac{\mathrm{N}}{2 \mathrm{~N}+1} \frac{1+2(2 \mathrm{~N}+1)^{2}}{\mathrm{~N}-1+3(2 \mathrm{~N}+1)^{2}}}
$$

The diagram of this relation is presented on the Fig. 2. 


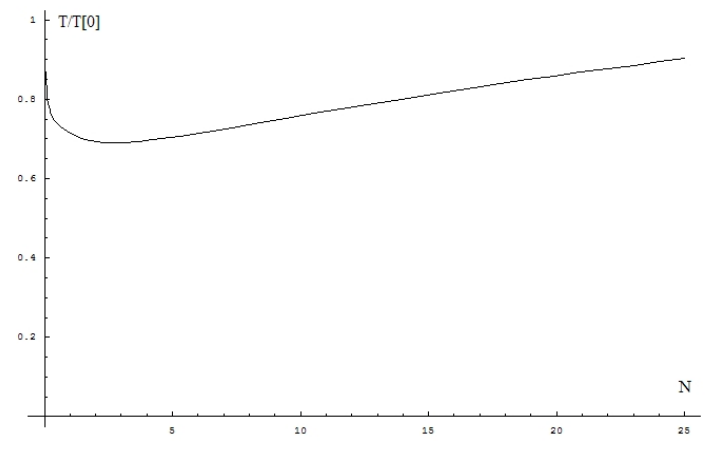

Figure 1. Relation between temperature and number of particles for the gravitonquintessence gas. Minimal value of temperature is obtained for $N_{0} \approx 2.73793853$ and is equal to $\frac{T\left[N_{0}\right]}{T[0]} \approx 0.69058084$.

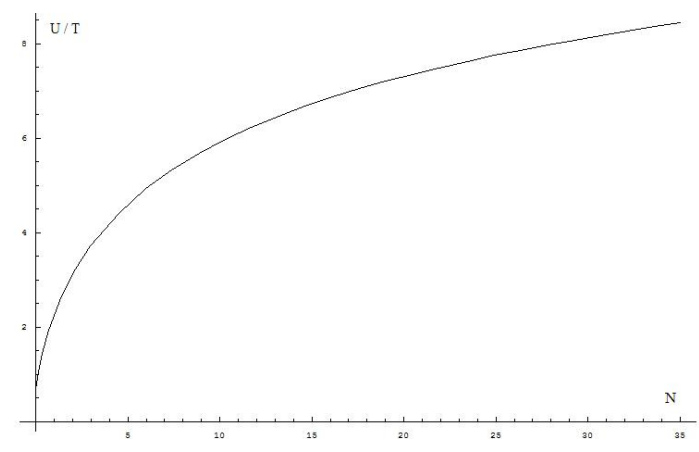

Figure 2. The equation of state for the graviton-matter gas in case of quintessence.

\section{Graviton-matter gas as solution for quantum gravity}

In this paper we have considered the Friedmann-Lemaitre model of the Universe with the quintessence. We have proposed the quantization procedure for this classical cosmological model in terms of the graviton-matter gas. As a result we have obtained nontrivial formulation of cosmology in terms of collective phenomena.

Physical meaning of the graviton-matter approach to the cosmic microwave background radiation temperature anisotropies arises from the following scenario. From the physical viewpoint we can think about our Universe as a gas of gravitons, gauge bosons, and material particles as electrons, quarks, Higgs particles etc. If in our thinking huge volume of the Universe is taken into account, the conclusion is that during our all observations and measurements of the Universe physical properties, we are on the position of an element of the gas - an observer in the Universe is an element of the Universe. By this way observations of the temperature anisotropies, understood as an effect of condensation of all particles and fields in the Universe, are natural conceptual consequence of this approach. From the graviton-matter gas viewpoint the quantum gravity has a meaning of effective theory and collective phenomena language seems adequate to description of the Universe physics. For this reason, in our opinion, the graviton-matter gas approach is interesting for further research in quantum cosmology.

\section{Acknowledgements}

I am especially thankful to Victor N. Pervushin and Andrey B. Arbuzov and for critical and develop remarks about my results. I am grateful to Andrew Beckwith and Michel Vittot for their interest in my solutions for quantum gravity.

\section{References}

[1] Mukhanov V., Physical foundations of cosmology, Cambridge University Press, Cambridge, 2005.

[2] Bojowald M., Universe scenarios from loop quantum cosmology, Ann. Phys. 15 (2006), 326-341, astro-ph/0511557.

[3] Glinka L.A., Pervushin V.N., Hamiltonian unification of general relativity and standard model, Concepts Phys., submitted, arXiv:0705.0655. 
[4] Glinka L.A., Pervushin V.N., Higgs particle mass in cosmology, talk on the Tenth European Meeting: From the Planck Scale to the Electroweak Scale, http://www.fuw.edu.pl/ susy/Planck07.html.

[5] Zakharov A.F., Zakharova A.A., Pervushin V.N., Conformal cosmological model test with distant SNIa data, astro-ph/0611657.

[6] Dirac P.A.M., Fixation of coordinates in the Hamiltonian theory of gravitation, Phys. Rev. 114 (1959), 924-930.

Dirac P.A.M., The theory of gravitation in Hamiltonian form, Proc. Roy. Soc. Lond. A 246 (1958), 333-343.

Dirac P.A.M., Generalized Hamiltonian dynamics, Proc. Roy. Soc. Lond. A 246 (1958), 326-332.

Dirac P.A.M., Generalized Hamiltonian dynamics, Can. J. Math. 2 (1950), 129-148.

[7] Einstein A., The meaning of relativity, Pricenton University Press, Princeton 1922.

Einstein A., A generalized theory of gravitation, Rev. Mod. Phys. 20 (1948), 35-39.

[8] Friedmann A.A., Über die Krümmung des Raumes, Z. Phys. 10 (1922), 377-386.

Friedmann A.A., Über die Möglichkeit einer Welt mit konstanter negativer Krümmung des Raumes, Z. Phys. 21 (1924), 326-332.

[9] Lemaître G.-H., l'Univers en expansion, Annales Soc. Sci. Brux. A 53 (1933), 51-85.

[10] Zelmanov A.L., Orthometric form of monad formalism and its relations to chronometric invariants and kinemetric invariants, Doklady Acad. Nauk USSR 227 (1976), no. 1, 78-81.

[11] Barbashov B.M., Pervushin V.N., Zakharov A.F., Zinchuk V.A., Hamiltonian cosmological perturbation theory, Phys. Lett. B 633 (2006), 458-462, hep-th/0501242.

[12] Hilbert D., Die Grundlagen der Physik, Gott. Nachr., 27 (1915), 395-407.

[13] Misner Ch.W., Thorne K.S., Wheeler J.A., Gravitation, Freeman and Company, San Francisco, 1973.

[14] Weinberg S., Gravitation and cosmology. Principles and applications of the general theory of relativity, John Wiley \& Sons, New York, 1972.

[15] Kolb E.W., Turner M.S., The early Universe, Addison-Wesley Publishing Company, 1988.

[16] Wheeler J.A., Superspace and the nature of quantum geometrodynamics, in Battelle Rencontres: 1967 Lectures in Mathematics and Physics, Editors C.M. DeWitt and J.A. Wheeler, New York, 1968, $242-307$.

[17] DeWitt B.S., Quantum theory of gravity. I. The canonical theory, Phys. Rev. 160 (1967), 1113-1148.

[18] Peskin M.E., Schröder D.V., Introduction to quantum field theory, Addison-Wesley, 1995.

[19] Bogoliubov N.N., Logunov A.A., Oksak A.I., Todorov I.T., General principles of quantum field theory, Fizmatlit, Moscow, 2006 (in Russian).

[20] Białynicki-Birula I., Białynicka-Birula Z., Quantum electrodynamics, Pergamon, Oxford, 1975.

[21] Pervushin V.N., Zinchuk V.A., Bogoliubov's integrals of motion in quantum cosmology and gravity, Phys. At. Nucl. 70, (2007), 593-600.

[22] Blaizot J.-P., Ripka G., Quantum theory of finite systems, Massachusetts Institute of Technology Press, 1986.

[23] Breuer H.-P., Petruccione F., The theory of open quantum systems, Oxford University Press, Oxford, 2002.

[24] Zubarev D.N., Morozov V.G., Röpke G., Statistical mechanics of nonequilibrium processes, Fizmatlit, Moscow, 2002 (in Russian).

[25] Alber G., Beth T., Horodecki M., Horodecki P., Horodecki R., Rötteler M., Weinfurter H., Werner R., Zeilinger A., Quantum information. An introduction to basic theoretical concepts and experiments, SpringerVerlag, Berlin - Heidelberg, 2001. 